\section{FEATURE ARTICLE \\ Human Functional Neuroimaging of Brain Changes Associated with Practice}

The discovery that experience-driven changes in the human brain can occur from a neural to a cortical level throughout the lifespan has stimulated a proliferation of research into how neural function changes in response to experience, enabled by neuroimaging methods such as positron emission tomography and functional magnetic resonance imaging. Studies attempt to characterize these changes by examining how practice on a task affects the functional anatomy underlying performance. Results are incongruous, including patterns of increases, decreases and functional reorganization of regional activations. Following an extensive review of the practice-effects literature, we distinguish a number of factors affecting the pattern of practice effects observed, including the effects of task domain, changes at the level of behavioural and cognitive processes, the time-window of imaging and practice, and of a number of other influences and miscellaneous confounding factors. We make a novel distinction between patterns of reorganization and redistribution as effects of task practice on brain activation, and emphasize the need for careful attention to practicerelated changes occurring on the behavioural, cognitive and neural levels of analysis. Finally, we suggest that functional and effective connectivity analyses may make important contributions to our understanding of changes in functional anatomy occurring as a result of practice on tasks.

Keywords: cortical plasticity, functional and effective connectivity, learning, neuroimaging/fMRI, practice effects

\section{Introduction}

Research within the last two decades has revealed that the functional properties of neurons within the central nervous system, as well as the neural circuitry within different brain areas, retain a significant degree of plasticity into adulthood (Buonomano and Merzenich, 1998). Research with both animal models and humans has shown that changes in neural representations can be induced not only in response to lesions of input or output pathways, but that the organization of the adult cerebral cortex can change substantially as a result of practice and experience (Karni et al., 1998; Kolb and Wishaw, 1998). Moreover, this research has shown that both developmental change and changes in response to experience can occur at multiple levels of the central nervous system, from changes at the molecular or synaptic level, to changes in cortical maps and large-scale neural networks (Buonomano and Merzenich, 1998). These discoveries challenge us to investigate how it is that the brain changes in response to experience. Modern neuroimaging methods such as positron emission tomography (PET) and functional magnetic resonance imaging (fMRI) are excellent tools in this endeavour, enabling the examination of how the
A. M. Clare Kelly ${ }^{1}$ and Hugh Garavan ${ }^{1,2}$

${ }^{1}$ Department of Psychology and Trinity College Institute of Neuroscience, Trinity College, Dublin, Ireland and ${ }^{2}$ Department of Psychiatry and Behavioral Medicine, Medical College of Wisconsin, Milwaukee, WI, USA

brain changes in response to practice or repeated exposure to a particular task.

Research aimed at understanding cortical plasticity and the principles governing experience-dependent plastic change in the brain is necessary in order to develop our understanding of not only the neural mechanisms of cortical reorganization but also the nature of cortical processing itself (Buonomano and Merzenich, 1998). The importance of this research however, goes beyond potential contributions to theories of learning and memory. Knowledge of how the brain responds to practice and experience is critical as the neuroanatomical, neurochemical and functional changes that take place may also underlie the recovery of function following damage to the brain. This knowledge will aid in our understanding of the mechanisms of repair and recovery in damaged or malfunctioning brains and how best to facilitate this rehabilitation through cognitive and behavioural interventions.

The literature dealing with the effect of practice on the functional anatomy of task performance is extensive and complex, comprising a wide range of papers from disparate research perspectives. Researchers have employed a variety of tasks and paradigms, and of practice techniques and schedules, and there is a corresponding variety in the pattern of results reported. Different studies report increased or expanded activations, decreased activations, or a reorganization of the functional activations underlying task performance. The current paper aims to provide an organizing framework that will guide understanding and interpretation of these results and thus resolve some of the apparent inconsistencies that exist in the literature. By identifying the factors that determine how the brain changes in response to task practice, this review will also suggest avenues for future research that will at once test the framework put forward in this review, and further our understanding of how the brain responds to practice and repeated task experience.

\section{Patterns of Practice-related Activation Change}

A growing number of human functional neuroimaging studies are investigating the changes in brain activation that occur as a result of practice on a range of motor, visuomotor, perceptual and cognitive tasks. Across studies, three main patterns of practice-related activation change can be distinguished. Practice may result in an increase or a decrease in activation in the brain areas involved in task performance, or it may produce a functional reorganization of brain activity, which is a combined pattern of activation increases and decreases across a number of brain areas.

There are a several different suggestions regarding what changes in neurological and psychological processes are 
reflected in practice-related activation change. Jonides (2004) proposes two possible cognitive consequences of practice greater skill at applying the initial strategy, or the development of a new strategy. At the physiological level, increased neural efficiency is suggested to be the main mechanism underlying the former type of plastic change, whereas cortical functional reorganization is suggested to underlie the latter. A number of neural mechanisms have been suggested as the basis for increased efficiency and cortical reorganization: the strengthening of existing synapses, the 'unmasking' of existing lateral connections through a change in the local balance of excitation and inhibition, formation of new synapses, changes in neuronal processes (such as dendritic length or spine density), or a combination of these (Rioult-Pedotti et al., 1998; Sanes and Donoghue, 2000; Schlaug, 2001; Kolb and Gibb, 2002; Munte et al., 2002). A caveat, however, is that little is known about the effect plastic changes in the brain have on functional imaging signals and it is unlikely that these different mechanisms can be differentiated on the basis of fMRI or PET data alone. This problem is amplified by the fact that the signals measured by PET and fMRI are indirect measures of synaptic activity (Poldrack, 2000). Relating specific changes in imaging signals to particular neurobiological mechanisms of plasticity is therefore problematic. Nonetheless, in the present paper we seek to demonstrate how our understanding of the relationship between task practice and neurophysiological change can be developed through a careful identification of the effects of task practice at each level of analysis - from the behavioural, cognitive and psychological levels, to the level of neuronal function.

\section{Activation Decreases}

Decreases in the extent or intensity of activations are common to the majority of studies examining task practice. The primary mechanism proposed to underlie activation decreases is increased neural efficiency. This increased efficiency may correspond to a sharpening of the response in a particular neural network so that only a minority of neurons now fire strongly in response to a particular task or stimulus (Poldrack, 2000). Practice-related activation decreases are therefore the result of more efficient use of specific 'neuronal circuits', learning at the Hebbian synapse being an example (Petersen et al., 1998). Decreases in activation therefore represent a contraction of the neural representation of the stimulus (Poldrack, 2000) or a more precise functional circuit (Garavan et al., 2000). In topographically organized cortex this will be seen as a reduction in the spatial extent of activation, or, in areas with distributed representations, as a reduction in activation (Poldrack, 2000).

\section{Activation Increases}

The term 'increases in activation' refers to both practice-related expansions in cortical representations and increases in the strength of activations. The lack of distinction between these two is based on the possibility that expanded representations are undetectable at the spatial resolution of most studies, or may be masked by spatial smoothing analytic methods. As a result, expanded representations may instead be reflected in increased strength of activations (Poldrack, 2000). On a neural level, increases in activation are suggested to reflect recruitment of additional cortical units with practice, seen in topographically organized cortex as an increase in the spatial extent of activation, or a strengthening of response within a region, observed as an increase in the level of activity within that region (Poldrack, 2000).

\section{Functional Reorganization of Activation}

We propose that practice-related reorganization of the functional anatomy of task performance may also be distinguished into two types, one constituting a redistribution, the other a 'true' reorganization. Both of these constitute some combination of activation increases and decreases and therefore to some degree are subserved by the same neuronal mechanisms as those discussed above. However, there is added complexity caused by variations in the form of those increases and decreases.

We consider the first of these patterns a pseudo-reorganization, or redistribution of functional activations. It constitutes a combination of increases and decreases in activation such that the task activation map generally contains the same areas at the end as at the beginning of practice, but the levels of activation within those areas have changed. The functional anatomy of the task therefore remains basically the same but the contribution of specific areas to task performance changes as a result of practice. This pattern may be considered a result of a practicerelated 'pruning' of functional activations and refers to the pattern of activation change observed when practice is associated with the attainment of automatic or asymptotic performance, and therefore a decreased demand on control or attentional processes and an increased demand on storage and processing in task-specific areas. It has been discussed previously by Petersen et al. (1998) in terms of a 'scaffoldingstorage' framework. According to this framework, a 'scaffolding' set of regions is used to cope with novel demands during unskilled, effortful performance. After practice, processes or associations are more efficiently stored and accessed and the scaffolding network falls away, evinced by decreased activation in those 'scaffolding' attentional and control areas. A coordinated increase in activation is observed in those areas underlying task-specific processes. Activations seen earlier in practice therefore involve generic attentional and control areas prefrontal cortex (PFC), anterior cingulate cortex (ACC) and posterior parietal cortex (PPC) are the main areas considered to perform the 'scaffolding' role, consistent with theories of PFC function and the involvement of these areas in the distributed working memory system. On the other hand, increases associated with highly practised performance are primarily seen in task-specific areas such as representational cortex - primary and secondary sensory or motor cortex, or in areas related to the storage of those representations, such as parietal or temporal cortex.

The second type of functional reorganization is considered here to reflect a 'true' reorganization of activations. It is observed as a change in the location of activations and is associated with a shift in the cognitive processes underlying task performance. This shift ('process switching': see Poldrack, 2000) means that neurobiologically and cognitively, different tasks are being performed at the beginning and end of practice, resulting in a coordinated increase and decrease of activation in separate brain regions, and is consistent with studies demonstrating a reorganization of activation as a result of explicit shifts or differences in task strategy (e.g. Bernstein et al., 2002; Glabus et al., 2003). Reduced activity in a particular region reflects less engagement of a particular cognitive process and increased activation reflects the engagement of an alternative system or 
the development of new representations or processes (Poldrack, 2000).

The distinction between the two patterns of activation change, redistribution and reorganization may be subtle, but we believe that it has important consequences for our understanding of practice effects. Two criteria are proposed to distinguish the two. First is that the key to redistribution of functional activations is the neurophysiological 'pruning' (Ramsey et al., 2004) of attentional and control areas. Thus, while activations in task-specific areas may be present early in performance, activations in the scaffolding areas may not be present late in performance. In some cases, as will be illustrated below, activation in these attentional and control areas may return to baseline as a result of practice.

Discriminating true reorganization from redistribution may often prove challenging with empirical data: the vagaries of brain imaging and statistical thresholding may make it difficult to determine whether it is the case that a cortical region is not activated and therefore not involved in a task, or whether it has reduced in activity to baseline or just above baseline levels. To add further complexity to the discrimination, it is possible that the neurophysiological 'pruning' of attentional and control areas is common to almost all practice-effects studies. That is, during naïve performance of most tasks, there will be some degree of supporting activation in attentional and control areas ('scaffolding') that drops off as one becomes familiar with the task. The extent to which these activations play a role in task performance will be determined by the task domain, the complexity of the task and other such factors, as will be discussed below. We therefore propose that a second criterion critical to discriminating between redistribution and reorganization is the determination of whether the cognitive processes in operation early in practice persist until late in practice. If the cognitive processes are changed by practice such that practised task performance amounts to performance of a cognitively different task, this is likely to be reflected in a neurobiologically different task map and therefore a true reorganization of functional activations. On the other hand, if the cognitive operations underlying task performance are not fundamentally changed by practice, but rather the amount of control and attentional support is altered, then the pattern of activation changes observed is likely to be that of a redistribution.

In Table 1 we have attempted to summarize some of the significant studies in this area and to illustrate the primary pattern of practice-related activation change observed in those studies. Table 1 may act as a reference for the reader to facilitate understanding of the discussion of these patterns of activation change, and of the individual studies when they are reviewed below.

\section{The Practice Effects Literature}

In consideration of the different patterns of activation change outlined above, and through a careful reading of the practice effects literature, we propose that there are a number of factors that must be taken into account in the discussion of practiceeffects research. Attention to these factors can explain the particular pattern of practice-related changes observed, and distinguish between the mechanisms likely to underlie those changes in activation. Below we discuss each of these factors in turn, illustrating their influence using examples from the literature. It will be clear to the reader that, rather than acting in isolation, the factors are interactive and any number of them can come into play in determining the particular pattern of practice effects observed in each study.

\section{The Effect of Practice on Task Processes}

The effects of practice at a cognitive level must be carefully considered in the interpretation of any study as the changes in cognitive processes underlying task performance can be a key to understanding the changes in functional activations observed. Meegan et al. (2004) drew attention to the necessity of this kind of task analysis in imaging research, emphasizing the need for a truly cognitive neuroscience. In our discussion of the patterns of redistribution and reorganization it is clear that one must establish which processes are involved in task performance and how these processes change with practice in order to make sense of the activation changes observed.

\section{Redistribution}

As suggested above, the pattern of redistribution of functional activations may be common to a number of studies as the neurophysiological 'pruning' of attentional and control ('scaffolding') areas may be a general result of increasing familiarity with the task. To review, brain activity is redistributed so that the task activation map remains more-or-less constant throughout practice but the levels of activation across the map change as a result of practice. Activations associated with highly practised, automatic or asymptotic task performance therefore tend to be task-specific, while activations early in practice also comprise generic attentional and control areas. Prefrontal cortex (PFC), anterior cingulate cortex (ACC) and posterior parietal cortex (PPC) are the main areas considered to perform the 'scaffolding' role.

Shadmehr and Holcomb (1997) used PET to examine brain activations during acquisition of a mechanical system imposed on rapid reaching movements by a robotic arm force field. Practice resulted in task performance becoming highly skilled and converging on that of a null field condition. A redistribution of activations from frontal cortex to posterior (parietal cortex, cerebellum) areas was only observed during a recall session that took place $5.5 \mathrm{~h}$ after the last practice session. Interestingly, these changes occurred in the absence of any further performance changes. The authors propose that this is consistent with the notion that acquisition of skilled movement is mediated through PFC structures and with time and practice, and as automation occurs, motor structures such as the cerebellum assume a greater role and possibly become the site of motor memory. [While the present studies are discussed in terms of redistribution of activations, there is some evidence that there may be a shift, or reorganization of the activations within the cerebellum as learning progresses (Nezafat et al. 2001; Doyon et al. 2002). The specific functional role of these activations awaits further investigation. However, this within-area reorganization does not discount our distinction between redistribution and reorganisation.] The results of this study are also consistent with those of Seidler et al. (2002) who saw learningrelated activations in premotor, motor, prefrontal, cingulate and parietal cortices, and in the thalamus, during practice on a serial reaction time (SRT) task. Activation in these areas was observed to dissipate with practice even though a distractor task prevented the behavioural expression of learning. On the other hand, cerebellar activation increased during the expression of performance improvements, once the distractor had been 


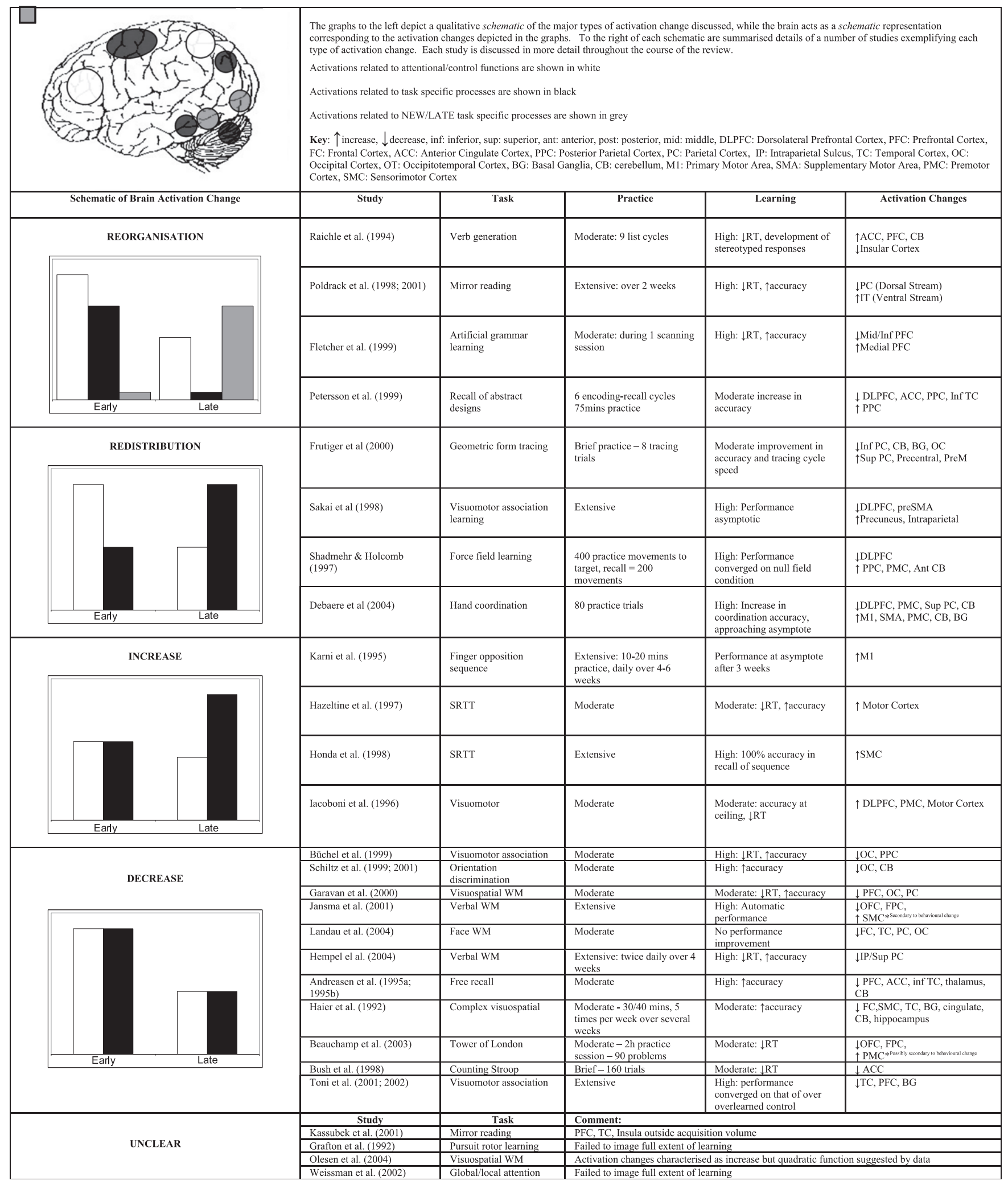


removed. Both these studies demonstrate the 'scaffolding' role of frontal and parietal areas, activation which is subject to 'pruning' as performance improves and becomes increasingly reliant on task-specific, performance-related areas located posteriorly in the brain. Furthermore, they demonstrate how learning-related changes in activation may occur in the absence of performance change, emphasising the complexity of the brain's response to repeated task experience.

Frutiger et al. (2000) had subjects practise a sequence of movements (tracing a geometric form). A pattern of increased activity in superior parietal and motor regions, and decreased activity in inferior parietal, cerebellar, striatal and occipital regions was associated with improved performance, suggesting that improvement on the task was due to increased activation of task-related neural systems and decreased activation of those implicated in attention, retrieval and monitoring. Similarly, Debaere et al. (2004) examined practice-related changes in activation during learning of a bimanual skill, requiring coordination of hand movements. Comparing initial learning to practised performance revealed learning-related decreases in right dorsolateral prefrontal cortex, right premotor, bilateral superior parietal cortex and left cerebellum. Conversely, learning-related increases in activation were observed in predominantly motor areas including M1, cingulate motor cortex, left premotor cortex, cerebellum and basal ganglia.

Sakai et al. (1998) imaged subjects while they learned the correct order of pressing two buttons sequentially in response to 10 pairs of targets until performance became automatic. They saw a transition of activations from left dorsolateral PFC and preSMA during early and intermediate stages to the precuneus and intraparietal sulcus in later stages. This redistribution of activations was seen as a learning-related transition from attention-demanding declarative processes to more automatic, procedural processes, consistent with the neurophysiological 'pruning' of attentional and control processes. Furthermore, good performers showed the decrease in prefrontal activity more quickly than poorer performers, who may have required more prolonged attentional support in order to perform the task. Congruent with this and the other studies discussed above, further studies (e.g. Hikosaka et al., 1998, 1999, 2002) have suggested crucial roles for the basal ganglia in motor learning (early activation) and the cerebellum in the behavioural expression of learned performance (late activation).

Jueptner et al. (1997) also demonstrated the neurophysiological pruning effect by showing that when subjects were instructed to reattend to the performance of an overlearned motor task, there was reactivation of prefrontal areas responsible for attentional control. Finally, redistributed activation relating to the role of attention and control during task performance has been observed in tasks comparing explicit and implicit learning. Grafton et al. (1995) and Doyon et al. (1996) both employed the SRT task, which requires subjects to respond with a key press to a series of stimuli (spatial locations; colours or numbers) presented in either random order or as a fixed, embedded sequence that cycles continuously. Learning on the SRT task, in the form of decreased RT and increased accuracy, can occur without awareness of this sequence; however, as a result of repeated exposure to the task, participants can gain explicit knowledge of an embedded sequence. Practice on the SRT task was observed to result in moderate improvements in task performance, and an increase in rCBF (measured with PET) in contralateral motor areas was associated with implicit learning.
Further to this, Grafton et al. (1995) observed that practicerelated explicit awareness of the sequence produced increases in fronto-parietal areas. Similarly, Doyon et al. (1996) observed mid ventrolateral frontal activity associated with explicit knowledge of the sequence. These studies demonstrate the components of the pattern of redistribution as they show the association between explicit attention to task performance and activations in prefrontal and parietal attentional areas, and the association between implicit or procedural task performance and increased activations in task-specific motor areas. Importantly, these studies also emphasize how an analysis of changes occurring on a cognitive and behavioural level as a result of task practice is necessary in order to interpret the associated changes in activation.

\section{Reorganization}

With practice and as performance becomes more skilled there may be a shift in the processes underlying task performance. As outlined above, this shift ('process switching') is associated with a true reorganization of the functional activations underlying task performance - the functional neuroanatomy and cognitive processes present early in practice are replaced by different processes and different neuroanatomy late in practice.

Raichle et al. (1994) provide the clearest example of how a change in task processes produces a functional reorganization of activations. Using PET, they imaged brain activity while subjects generated verbs to a list of nouns. The generate task was repeated for several blocks ( $\sim 15$ min practice) and subjects were then scanned again while they generated verbs to both the original list and a new list of nouns. Practice significantly reduced RT and also led to the development of stereotyped responses to $\sim 90 \%$ of the nouns. Raichle et al. observed a reorganization of functional anatomy underlying the task reduced activation in ACC, left PFC and right cerebellum, and increased activity in sylvian-insular cortex. Underlying this reorganization was a practice-related change in task processes - there was a switch away from effortful, semantic processing and selection from an unlimited set of responses to paired-associate episodic recall, minimizing effort and semantic processing as well as the set of possible responses. In support of this analysis, when a new word list was introduced, the authors observed reactivation of the frontal areas and decreased activation of left sylvian-insular cortex.

The reorganization of functional activations associated with practice-related changes in task processes has been observed in a number of other studies. Poldrack and co-workers (Poldrack et al., 1998; Poldrack and Gabrieli, 2001), in a series of fMRI studies into the neural basis of mirror reading, explicitly took into account the fact that practice produces a change in the nature of processing involved in task performance. Mirror reading requires visuospatial transformation early in learning, which gives way to object recognition late in learning, as performance becomes automatic. Subjects were imaged while they made lexical decisions on mirror-reversed stimuli prior to and following extensive training (over 2 weeks). The results revealed that the practice-related shift in task processes was associated with a reorganization of functional anatomy underlying mirror reading with decreased activation in the dorsal visual stream (visuospatial) and increased activation of the ventral stream (object recognition).

A further example is a study by Fletcher et al.(1999), who, in an artificial grammar-learning paradigm, observed a reorganization 
of neural activity underlying task performance from that associated with learning the grammatical status of individual items (episodic learning - right middle/inferior PFC) to that associated with learning the rules of the grammar system (implicit, semantic learning - left medial PFC). An effective connectivity analysis supported the transition, revealing corresponding increases and decreases in the strength of frontoparietal and intra-prefrontal connections associated with rule learning and episodic memory retrieval.

Petersson et al. (1999) also observed a reorganization of functional activations. Using PET, they compared practised to novel free recall of abstract designs. While they observed decreases in activation associated with decreased reliance on attentional and working memory processes (PFC, ACC, PPC), and increases in areas related to task-irrelevant processing (auditory cortex, insula), they also saw reorganization of activations relating to the transition from reliance on non-consolidated representations (inferior temporal cortex) to the recall of more developed representations of the abstract designs (occipitotemproal region). In other words, a reorganization of activations was observed as the task processes shifted from effortful, working memory-based recall to those involving more automatic recall of consolidated representations of the abstract designs.

These studies demonstrate very clearly how an understanding of the effects of practice on the cognitive operations underlying task performance is necessary in order to make sense of the shift in functional activations. One point to note is that here we have identified the Raichle et al. (1994) study as a reorganization, while Petersen et al. (1998) discussed it in terms of the scaffolding-storage network, which we have associated with the pattern of redistribution. This divergence highlights the usefulness of our distinction between reorganization and redistribution, and the necessity of a careful task analysis in the understanding of one's pattern of results, as neither Petersen et al. nor Raichle et al. explicitly identified the switch in task processes that occurred in the verb generation task as a result of practise and therefore did not interpret the reorganization of activations in terms of this process switch.

\section{The Effect of Task Domain}

When the cognitive processes involved in task performance are not substantially altered, even after extensive practice or changes in behavioural output, then increases or decreases in activation, rather than a reorganization, are observed. These quantitative changes reflect plastic changes within the functional network underlying task performance. Studies demonstrating these conflicting patterns of quantitative change may be differentiated on the basis of task domain. Schiltz et al. (2001) have outlined the importance of distinguishing between highlevel cognitive skills and low-level visuomotor and perceptual skills. There are a number of factors contributing to such a distinction in practice effects between task domains. Of primary importance is the possibility that the functional and structural cortical organizations underlying performance in different task domains are associated with divergent plastic responses.

A number of researchers suggest that there is a particular signature of neural change associated with practice in primary motor or sensory cortex (e.g. Hikosaka et al., 1998; Karni et al., 1998; Hikosaka et al., 2002; Ungerleider et al., 2002; Korman et al., 2003; Kleim et al., 2004). These authors propose that motor learning occurs in several phases: a fast initial phase of performance gains, which occurs over initial trials and corresponds to the acquisition of a task-relevant routine. This is followed by a consolidation period lasting several hours. Finally, there is a slow learning phase requiring thousands of training trials, which changes the strength of links between task-related sensory or motor units and leads to gradual increases in performance (Schiltz et al., 2001; Munte et al., 2002). The phases of motor learning can be associated with specific activation changes. During the initial phase of learning there are often increases in attentional and control areas, as discussed above in the section on redistribution. Importantly, however, the final, slow learning phase is typically associated with expansions or increases in activation in motor areas of the brain (e.g. Schiltz et al., 2001; Ungerleider et al., 2002).

It is suggested therefore that increased levels of activation or expansions in the area of activated cortex are the result of changes in highly specific task-related cortical representations that are particular to topographically organized motor and sensory cortex (although an exception to this may be visual cortex: see Schiltz et al., 1999). (According to Schiltz et al. (2001), studies of perceptual learning have observed activation decreases more consistent with studies of higher cognitive skill learning. They suggest that this is because visual cortex is not topographically organized (unlike auditory cortex or motor cortex) and therefore may depend on different plastic mechanisms resulting in activation decreases. One suggestion is that the decreases correspond to decreases in the receptive field of the neurons in response to training on highly specific perceptual discriminations. Training of such discriminations in auditory and somatosensory modalities, on the other hand, has been shown to expand the bandwidth or receptive field of neurons (Munte et al., 2002).] Mechanisms of plasticity in association or non-primary cortex may differ, however, thus the patterns of activation change associated with practice on tasks primarily subserved by these regions of cortex may also differ. We suggest that the predominant pattern of practice-related change in association cortex is a decrease in activation across the network of brain areas underlying task performance.

In support of this conjecture, Kolb and Gibb (2002) suggest that experience-driven change in prefrontal cortex is different from that in other cortical areas. This is based on findings of differential experience-related effects in prefrontal cortical neurons when compared to parietal and occipital cortical neurons. Neurons in parietal and occipital cortex showed changes in dendritic length, whereas neurons in prefrontal cortex showed increased spine density. Furthermore, the authors observed that exposure to psychomotor stimulants, certain neurotrophic factors and gonadal hormones had divergent effects on prefrontal neurons compared to motor or sensory neurons. The implication of these differences, the authors suggest, is that synaptic changes related to the performance of tasks that engage prefrontal cortex may not be the same as those that are found in motor or visual cortex following learning on motor or visual tasks. We suggest that this may be a basis for differential activation changes associated with sensory/motor and higher cognitive tasks, though whether we are capable of identifying these changes on the micro, neuronal level, by means of our investigations at the macro level using fMRI or PET is, at present, unclear.

Nevertheless, there are other differences between task domains that are more likely to be observed at a macro level. Primarily, there may be divergent practice-driven changes 
related to the representations contained in different areas of cortex. Structural, anatomical and electrophysiological studies show that the representation area of primary cortices depends on practice and adapts to current needs and experiences, suggesting, for example, that long-term motor training should induce an extension of the representation area of primary cortices (Hund-Georgiadis and von Cramon, 1999). Modifications in horizontal connectivity in primary cortex are thought to be one of the main mechanisms through which this expansion occurs. Buonomano and Merzenich (1998) assert that horizontal connections are of particular importance in topographic cortex as experience-driven changes in neuronal receptive fields and other emergent response properties may rely on connections from neighbouring cortical sectors. Modifications in horizontal connectivity in primary cortex are proposed to result from changes in synaptic efficacy, possibly through longterm potentiation-like mechanisms and are also suggested to underlie experience-related plasticity in other, non-motor cortex, e.g. visual cortex (Hund-Georgiadis and von Cramon, 1999). Furthermore, the fact that changes in horizontal connectivity may be the basis of practice-related changes in primary cortex is concordant with the observation that experience produces increases in dendritic length in posterior cortex, as discussed above (Kolb and Gibb, 2002). On a cognitive level, these practice-related changes in representations have been associated with the development of a specific memory trace an 'internal model' (Shadmehr and Holcomb, 1997) of the practised task. Thus, we suggest that in sensory and motor tasks, which involve topographical cortical representations, a primary outcome of practice is an expanded representation within primary cortex resulting from increased connectivity within that area. This practice-related change is associated with specific mechanisms of synaptic plasticity, primarily modification in horizontal connections, and is observed as an increase in activation or an expansion in the area of activation.

In contrast, we suggest that the representational demands of higher-level cognitive tasks are such that a more distributed network of brain regions is observed to underlie task performance. The specific operations and stimuli involved in the performance of cognitive tasks may not therefore lend themselves to the formation of the kind of long-term localized and specific memory trace hypothesised to exist for sensory and motor tasks. The demonstration that areas of association cortex, PFC in particular, contain neurons showing the flexible coding of information and the formation of malleable or transient representations (e.g. Rainer et al., 1998; Duncan, 2001; Miller and Cohen, 2001) supports this conjecture. Instead, we suggest that practice on cognitive tasks increases the efficiency of the distributed task network and that this increased efficiency is observed as changes in connectivity between nodes of the network and decreases within the separate areas as those areas become more efficient at performing their particular function. That practice-related decreases in neuronal activity are observed in tasks primarily demanding PFC is consistent with neurophysiological research demonstrating decreased activity in the neurons of PFC of monkeys with learning (e.g. Chen and Wise, 1995; Asaad et al., 1998). By analogy, we suggest that whereas practice-related changes in activation on sensory/ motor tasks may predominantly take the form of increased connectivity within primary cortex, the type of plasticity and activation change associated with practice on higher cognitive tasks is a result of changes in connectivity between a more highly distributed network of functional areas. This suggestion is supported by studies showing changes in connectivity between regions as activity within those regions decreased as a result of learning (Buchel et al., 1999; McIntosh et al., 1999).

A caveat emerges from this proposal, however. It will be clear to the reader that there is a great deal of complexity involved in the attempt to study plastic changes associated with the practice of even simple behaviours. Authors such as Wolpaw (1997) have demonstrated how the repeated performance of even the simplest of behaviours, a reflex, can produce complex plastic changes at multiple sites in the central nervous system, including sites that do not appear to contribute to the learned behaviour itself. This raises questions as to our ability to make straightforward associations between activation changes and purported underlying mechanisms of plasticity, such as increased neural efficiency. Nonetheless, we emphasize that endeavouring to ensure thorough and informed examination of the effects of practice at each level of analysis, from the neural to the behavioural, should guide our understanding of how the brain changes in response to repeated task experience. Although this may be the best we can accomplish given our present level of technical sophistication, developments in highfield MRI, and the increasing use of complementary tools such as diffusion tensor imaging (DTI) may go some way towards reducing the complexity involved and increasing our confidence in our findings. These possibilities are discussed further below in the final section concerning the issues raised by and implications of this review.

Supporting the distinction we have drawn between higher cognitive and sensory/motor tasks based on their divergent representational properties is the fact that extensive practice on motor tasks typically produces highly specific improvement in the trained task only, and often for the trained effector only. That is, practice-related improvements in performance do not generalize to either the untrained effector, or to different task sequences or stimuli (e.g. Ungerleider et al., 2002; Korman et al., 2003). Only a small number of studies have examined the transfer of practice-related improvement in higher-level cognitive tasks, but the results of those studies are promising, showing significant transfer of practice-related improvement to similar cognitive tasks (Klingberg et al., 2002; Olesen et al., 2004). These findings await further empirical investigation and experimental support.

A final distinction between task domains involves the extent to which participants are practised or trained on the task of interest. Practice on motor tasks is typically extensive and conducted over considerable periods of time (weeks). Practice on cognitive tasks on the other hand, tends to be brief (hours), which may carry the implication that the full extent of practicerelated changes has not yet been examined in a cognitive task. This idea is elaborated below in the section considering the effect of the phase of learning imaged and the duration of task training.

In summary, we propose that there are differences in the types of practice-related change occurring in sensory/motor and higher-level cognitive tasks both at the level of mechanisms of neural plasticity, and at the level of cortical representations. Furthermore, we propose that analysis and understanding of the differences in practice-related regional activation change will be complemented by connectivity analyses. We hypothesize that practice on motor and sensory tasks will be predominantly associated with changes in connectivity within primary cortex 
or between motor/sensory areas. On the other hand, we suggest that practice on higher-level cognitive tasks will be associated with changes in connectivity between a larger number of more widespread and distributed brain areas.

The extant literature supports the task-domain differentiation. Practice-related increases in contralateral motor areas have been demonstrated in a number of studies, the most frequently cited of which is Karni et al. (1995), who examined activations in primary motor cortex while subjects performed a trained and untrained (control) four-part finger-to-thumb opposition sequence. The trained sequence was practised for 10-20 min each day over several weeks; the untrained sequence was performed only during scanning (once per week over 4-6 consecutive weeks). Performance on the trained task reached asymptote after 3 weeks, from which time it evoked an activation map that was consistently larger than that of the control. This larger activation map reflects an expansion of the trained sequence representation in $\mathrm{M} 1$. The authors suggest that practice induces recruitment of additional M1 units into a network specifically representing the trained sequence.

Other studies have observed practice-related increases in activation in motor cortex and have similarly attributed this to mechanisms of motor plasticity. The majority of these studies have already been discussed above, in the section on redistribution, as the typical pattern is that of initial activations in 'scaffolding' areas such as PFC, ACC and PPC, which attenuate relatively rapidly as a result of repeated task experience. These decreases are accompanied by increased activity in task-specific (i.e. motor) areas. For example, Debaere et al. (2004) observed learning-related increases in activation in predominantly motor areas including M1, cingulate motor cortex, left premotor cortex, cerebellum and basal ganglia during acquisition of a bimanual skill. Shadmehr and Holcomb (1997) also observed increases in motor structures such as the cerebellum associated with increased skill in a motor task. Similarly, studies by Doyon et al. (1996), Grafton et al. (1995), Hazeltine et al. (1997) and Honda et al. (1998) demonstrated increases in rCBF (measured by PET) in contralateral motor areas associated with practice and implicit learning in the SRT task. In all of these studies there were also decreases in control and attentional areas associated with the neuropsychological 'pruning' accompanying repeated task experience and improved performance. Note that in the case of the Karni et al. (1995) study, the acquisition volume did not include areas outside M1, precluding any conclusions concerning the activation of 'scaffolding' areas during practice. Nonetheless, it would be reasonable to assume that the pattern of activations observed would have been similar to that of the other studies, with early activations in prefrontal and parietal areas that quickly attenuated with practice.

Practice-related increases in primary cortex are thus relatively robust. Furthermore, these changes are consistent with studies showing expanded representations in topographic sensory and motor cortex resulting from extensive experience with a particular type of stimuli, for example, increased representations in somatosensory cortex for the dominant index finger of Braille readers compared to that of non-Braille readers (Pascual-Leone and Torres, 1993), for left-hand digits in string players compared to controls (Elbert et al., 1995); and in primary auditory cortex of musicians relative to non-musicians for piano tones (Pantev et al., 1998). Furthermore, in these and a number of other motor studies (e.g. Karni et al., 1995; Korman et al., 2003), once practice-related improvement was consoli- dated, there was no transfer of learning to either the unpracticed effector or to an untrained task sequence, emphasizing the specificity of the representations resulting from practice in motor tasks.

In contrast to the practice-related increases in activation observed in sensory and motor studies, practice on specifically cognitive tasks, such as those involving the learning of complex sensorimotor associations, free recall or working memory, not associated with a change in the cognitive operations involved in performance, produces activation decreases in the functional network underlying task performance.

Toni et al. (2001, 2002) imaged subjects while they learned to associate four patterns (line segments) with flexion of fingers on the right hand and compared learning-related activations to those observed in an overlearned control condition. As learning progressed, performance on the learning condition converged onto values of the prelearned control condition. There was learning-related activity in a distributed cortical network, centred on a tempero-prefrontal circuit, and the overlearned condition was associated with lower levels of activity in this circuit. Similarly, Büchel et al. (1999), imaged subjects while they learned and recalled an association between three sets of 10 simple line drawings and 10 spatial locations. With learning, decreases in activation in both dorsal and ventral visual pathways were seen, suggested by the authors to be the result of enhanced response selectivity. These authors also implemented an effective connectivity analysis to assess the hypothesis that this enhanced response selectivity was due to changes in connectivity within the system at a synaptic level. Consistent with this hypothesis, effective connectivity between dorsal and ventral visual pathways was found to increase over time, while connections within the dorsal pathway decreased. Furthermore, changes in effective connectivity occurred earlier in subjects who learned the associations faster. These findings are also consistent with Haier et al. (1992), who observed a widespread decrease in glucose metabolic rate (GMR) across cortical areas activated by a complex visuospatial/motor task following several weeks of moderate training. The decreases in GMR were correlated with improvements in performance, consistent with the hypothesis that decreases in activity reflect more efficient information processing in task-relevant areas, and suggesting that the brains of subjects doing the task well function more efficiently than poorer performers. This association is again supported by recent research with another complex task, the Tower of London problem. In their study, Beauchamp et al. (2003) observed reduced activations in prefrontal areas following practice and observed that these decreases were correlated with improved performance on the task. The relation between better task performance, decreased activation and neural efficiency is intriguing and merits further investigation as it may reveal how it is some brains perform better than others.

Practice-related decreases in activation were also seen by Andreasen et al. (1995a,b), who imaged the effects of practice on the brain activations associated with recall of complex narratives and word lists using PET. Comparing practised to novel free recall revealed decreased activation in the functional network underlying task performance - including PFC, ACC, inferior temporal cortex, thalamus and cerebellum, again suggesting practice permitted recall to be performed more efficiently. 
Decreased activations associated with practice on working memory tasks are also quite robust. Garavan et al. (2000) examined the effect of practice on a visuospatial workingmemory (VSWM) task on activation over two timescales: one fMRI scanning session, corresponding to moderate practice; and over three scans with intervening task training, corresponding to relatively extensive practice. RTs decreased with practice and accuracy varied as a function of task difficulty. The accuracy effect persevered to the end, demonstrating persistent involvement of VSWM, despite practice. Analysis of changes in activation occurring over a single scanning session (80 trials, 20 min) showed activation decreases in the majority of areas activated during performance of the task (prefrontal, parietal and occipital cortex), suggesting an increase in neural efficiency was underlying improved task performance. There was little further change in activation over the longer time period, leading the authors to conclude that practice resulted in a 'cleaner' functional map of the process involved in VSWM, as extraneous processes dropped off with practice, leaving the essential functional neuroanatomy of working memory. Similar activation decreases were observed by Jansma et al. (2001) following practice on a Sternberg task, Landau et al. (2004) following practice on a facial working memory task, and Hempel et al. (2004) following practice on the $n$-back task. There is consensus among these authors that the practice-related decreases reflect increased neural efficiency in the brain network underlying task performance - corresponding to more effective task processing and implementation of performance strategies. A further example is a study by Bush et al.(1998), who practised subjects on both neutral and interference trials of a counting version of the Stroop task. With practice they observed decreased activation in the ACC for interference trials, reflecting increasingly efficient interference resolution as a result of practice.

One exception to this pattern of results is a recent study by Olesen et al. (2004), who observed a practice-related increase in activation across a working memory network. However, Olsen et al. employed a paradigm in which task difficulty continually increased during practice by means of a performance-monitoring algorithm. As a result, despite extensive task practice, performance was not asymptotic, a factor that may explain the activation increases (this is discussed further in the next section). In addition, a number of studies have demonstrated how increased task difficulty or load is associated with increased BOLD activation (Rypma and D'Esposito, 1999; see also e.g. Jansma et al., 2000; Rypma et al., 2002; Druzgal and D'Esposito, 2003). Thus, even though participants were imaged during the performance of both high- and low-load conditions, it is possible that levels of activation remained artificially elevated as a result of the training paradigm. Furthermore, there is a suggestion that the pattern of activation changes observed by Olesen et al. (depicted in Fig. 3d) could be described by a quadratic function - by the final scanning session activation appears to be decreasing. This suggestion is based on a recent paper by Hempel et al. (2004) and is discussed further in the next section concerning the effect of the phase of learning imaged on the pattern of results observed.

\section{The Effect of the Extent of Practice, Level of Performance Attained and Imaging Time-window}

In the discussion of the effect of task domain above, attention was drawn to different phases or stages in the acquisition of motor or perceptual skill. These phases have been associated with different amounts of change on the level of primary cortical activation. Specifically, in motor studies, a single session or brief amount of practice can result in a diminution of the size of activation area in M1, while over longer periods of practice, the extent of activation in $\mathrm{M} 1$ is observed to increase, corresponding to the second phase of motor learning (e.g. Ungerleider et al., 2002). Similarly, in the discussion of the pattern of redistribution, we highlighted the association of practice-related decreases in attentional and control areas with the attainment of automatic or asymptotic performance. These factors demonstrate that the point in practice at which participants are imaged will have significant effects on the levels of activation observed. In order to draw strong conclusions regarding the effects of practice in any study, researchers must be sure they have imaged the entire window of practicerelated effects.

Illustrating this issue are studies in which training was brief, performance did not reach asymptote or in which the imaging schedule did not enable investigators to observe the full time course of learning. Such studies tend to find patterns of activation inconsistent with those discussed above, for example, showing continued high levels of activation in scaffolding and control areas.

For example, Grafton et al. (1992) practised subjects only briefly on a SRT task and saw general increases across the task network, contrary to the pattern of decreases in prefrontal and parietal control areas that would be expected. Iacoboni et al. (1996) also observed only increased activation during brief practice on a motor task. However, decreases in DLPFC were becoming evident at the end of practice, suggesting that these decreases would continue with more extensive practice, consistent with the redistribution pattern. Finally, Weissman et al. (2002), in practice on a global/local attentional cueing paradigm with incongruent and congruent stimuli, saw decreases in areas related to attentional orienting but increases in those related to conflict resolution on incongruent trials. These changes, however, were greatest over the earliest part of practice, particularly during the first half hour, suggesting that these may be related to novelty rather than practice - scanning did not extend past $1 \mathrm{~h}$ and it is possible that other changes may have occurred with further practice.

A recent study by Hempel et al. (2004) supports this analysis. They examined the effects of training on an $n$-back task over a period of 4 weeks. They observed initial increases in activation in task-specific areas followed by decreases in activation by the end of training. They suggest that decreases in activation values are associated with the consolidation of performance gains after extensive practice and conclude that training-related changes in activation follow an inverse U-shaped quadratic function, with initial increases followed by later decreases. As discussed above, this interpretation may apply to the data of Olesen et al. (2004), which, despite being characterized as demonstrating practice-related increases in activation, are suggestive of a quadratic function with a relative reduction in activation in the last scanning session compared to the penultimate one. This leads to the question of whether further decreases, and therefore an overall decrease in activation, would have been observed had further scans been carried out. The results of that study would then conform to the pattern predicted on the basis of the factors discussed above. 


\section{The Influence of Other Factors}

Finally, there are a number of further factors that can play a role in determining the pattern of practice-related changes in activation observed in any study. While these factors may act in isolation they also may have interactive effects with the main factors outlined above.

A very significant confound arises from practice-related changes in behavioural output. Changes in performance are an inherent characteristic of practice. Such changes include direct behavioural effects such as increased accuracy, decreased reaction time, increased processing speed and secondary taskrelated effects such as reduced errors, decreased time on task and more frequent responses. A confound arises here because it is possible that any changes in activation observed are secondary to the behavioural change, rather than the presumed underlying practice-driven changes in neural efficiency or cortical representations (Poldrack, 2000; Sanes and Donoghue, 2000). For example, van Mier et al. (1998) observed a practicerelated increase in M1 activation that is confounded by a concurrent increase in the velocity of motor output, which may in itself explain the increased M1 activity. Neural activation also differs as a function of duty cycle (time on task) or task pacing, which can also change as a result of practice. Some studies have controlled for this effect by having participants perform at a fixed, cued pace, and measuring practice-related performance improvement in terms of increases in accuracy (e.g. Karni et al., 1995; Jueptner et al., 1997; Toni et al., 1998). Finally, learning may also change the degree to which subjects are aware of the task or stimulus structure, which may result in a change in neural activity (e.g. Honda et al., 1998). The changes these variables can produce can be mistakenly identified as reflecting practice-related processes rather than consequences of those processes (see Poldrack, 2000, for an extensive discussion of these issues). It is imperative, therefore, that when interpreting activation changes one can be assured that those changes are the primary result of the hypothesized underlying neural plasticity, and not secondary to practicerelated changes in behavioural output. Careful and thorough task analysis will help ensure this end.

Another potential confound arises from those areas associated with task-irrelevant processing. These areas are proposed to be involved in 'default' brain processing and typically become deactivated during task performance, relative to a rest state (Raichle et al., 2001). Practice and increasing automaticity may lead to a decrease in the attentional suppression of irrelevant information processing, thereby decreasing the amount of deactivation in these task-irrelevant areas. Apparent increases in activation may therefore be spurious, a reflection of these reduced deactivations. While some researchers are aware of such effects (e.g. Petersson et al., 1999), difficulty identifying deactivations in imaging data means that this confound may arise more frequently than is acknowledged in the literature at present.

Another issue in some studies is the size of the acquisition volume. For example, Kassubek et al. (2001) scanned subjects using fMRI as they read either mirror inverted words or plain text before and after a training session. Reading of mirrorinverted items activated the dorsal visual pathway and premotor cortex, and a significant practice-related reduction in activation in these areas was observed. However, the acquisition volume did not include PFC, insular cortex or the temporal lobes. Thus, it is possible that the transition from the dorsal to the ventral visual processing stream, associated with a transition from visuospatial transformation to object recognition, as observed by Poldrack et al. (1998), was missed because the ventral stream was outside the field of view. It may also be for this reason that a pruning-related decrease in PFC activity was not observed. Similarly, in the Karni et al. (1995) study, only area M1 was imaged, again raising the possibility that there were practicerelated changes in other brain areas that were not observed.

Consideration of the previous experience of the participants with the task/stimuli being used is also important - a very different pattern of activation change may result in participants with a high level of familiarity compared to those naive with respect to the particular task or stimulus employed. This was demonstrated by Hund-Georgiadis and von Cramon (1999), in their comparison of highly skilled musicians and non-musicians during performance of a motor tapping task. Musicians activated a smaller neural network than non-musicians but also showed a practice-related increase in M1, while non-musicians did not. These results were taken to indicate that the effect of pre-practice experience was such that the activation pattern shown by the musicians seemed to resemble the later, slow learning phase of non-musicians, in which practice-related increases in M1 activation are typically observed (as demonstrated by, for example, Karni et al., 1995). Pre-practice experience may thus affect the speed of learning and the timing of the appearance of practice-related activation changes.

Pre-existing individual differences may also play an important role in the particular practice effects observed. The relation of factors such as better performance, speed of learning, or intelligence to practice-related changes in activation or connectivity has been demonstrated in a number of studies (e.g. Haier et al., 1992; Buchel et al., 1999; Beauchamp et al., 2003) and has been explicitly discussed in a recent paper by Neubauer et al. (2004). Contrary to our predictions of decreased activations as a result of practice and improved performance on higher level cognitive tasks, some authors have drawn attention to the association between higher intelligence, better performance and greater levels of activation, and have predicted practice-related activation increases in complex cognitive tasks (e.g. Olesen et al., 2004). Neubauer et al., however, assert that while higher levels of activation are observed early in practice in better or more intelligent participants, the negative correlation between intelligence or improved performance and activation emerges only after practice. Thus it is possible that 'better' brains learn faster and what is seen in the activation-performance/intelligence correlations is a manifestation of high activity underlying this more rapid learning. This is consistent with Büchel et al. (1999), who demonstrated a relationship between the onset of increased connectivity as a result of task practice (related to increased neural efficiency) and speed of learning, indicating that the brains of faster learners adapt to experience more rapidly than those of slower learners or poorer performers.

\section{Summary}

While an attempt has been made to offer a comprehensive review of the extant literature, there have inevitably been some omissions. [In particular, we have omitted discussion of the priming literature, despite the existence of a number of studies relating to practice effects (e.g. van Turennout et al., 2003), but for which it is more difficult to tease apart the effects of 
repeated task experience from repeated exposures to the task stimuli.] Nevertheless, the following summarizes the conclusions that can be drawn from this review:

- The pattern of practice-related changes seen in any study is explicable by reference to a number of factors that play a role in the task. These are: (i) The effect of practice on the cognitive processes involved in task performance. A reorganization of activations is associated with a change in cognitive processes. A redistribution of activations is associated with increased reliance on task- or process-specific regions and decreased reliance on control and attentional processes in areas such as PFC, ACC and PPC. (ii) The effect of task domain. Differential mechanisms of plasticity and representational modes between sensory/motor and cognitive domains mean that practice has a divergent effect on functional activations, increasing activity in sensory/motor tasks and decreasing activation in higher cognitive tasks. (iii) The effect of the time-window of imaging. The point in practice at which participants are imaged has significant effects on the levels of activation observed. In order to make strong conclusions regarding the effects of practice in any study, researchers must be sure they have imaged the entire window of practice-related effects. (iii) Further factors which have an effect include: the level of previous experience with the task, pre-existing individual differences, and confounding factors such as changes secondary to changes in behavioural output, task difficulty or load and default-state brain processing.

- Careful analysis of the practice-related changes occurring in processes at the cognitive and behavioural, as well as neurophysiological level is critical to the interpretation of the results of any practice effects study and an understanding of the influence of the above factors on the pattern of activation changes observed.

\section{Connectivity}

Throughout this review we have alluded to the fact that a better understanding of practice-related changes in the functional anatomy of task performance will be gained through the complementary analysis of practice-related changes in effective and functional connectivity as well as changes in regional activations. Already, a number of researchers have shown that brain connectivity changes with practice, as mentioned above. These studies (e.g. Buchel et al., 1999; Fletcher et al., 1999; McIntosh et al., 1999) demonstrate how decreased activation within a particular area may be associated with increased connectivity between that area and another, suggesting they are working together more efficiently to achieve the task. It is for this reason that we suggest that the investigation of practicerelated changes in connectivity can inform our interpretation of regional activations.

In order to clarify our emphasis on the benefits to be gained from an increased use of functional and effective connectivity analyses in the study of practice-related changes in brain function, a brief explanation of those techniques and their application is warranted (for an in-depth discussion, refer to McIntosh et al., 1994, 1996; Buchel and Friston, 1997, 2000; McIntosh, 1998, 1999; Friston, 2002).

It has become increasingly clear that the brain accomplishes successful behaviour by means of the concerted activity of a number of regions, rather than specific activity in any one functional area. It is because of the intense connectivity that exists between cells of the brain that the task of mapping cognition to the brain is so complex (McIntosh, 1999). In contrast to this, cognition is often viewed as the additive result of a large number of separable and localizable functions in the brain, a view that fails to take adequate account of this connectivity. McIntosh, however, has developed the idea of a 'neural context' (see e.g. McIntosh, 1998, 1999). This means that the function of a particular brain region should be viewed in terms of the emergent properties of large-scale neural network interactions - a single region may demonstrate the same pattern of activity across several behavioural and cognitive operations, but its interactions with other brain areas may differ across those operations. The cognitive operations performed by that area are therefore determined by its interactions with other related regions. 'The important factor is not that a particular event occurred at a particular site, but rather under what neural context did that event occur - in other words, what was the rest of the brain doing?' (McIntosh, 1998, p. 533).

The predominant analytic method within the practice effects literature is the examination of practice-related changes in regional activity. However, as McIntosh emphasizes, changes in neural interactions can be more informative than changes in regional activity. Areas showing strong interactions across different tasks may not also show consistent activation, or may not even be active relative to baseline. Activation analysis detects differences only while connectivity analyses can reveal changes in brain interactions not detectable though activation analysis (McIntosh, 1998). Connectivity analyses are important in revealing changes associated with task practice as they provide information about how localized regions work together as large-scale neural networks, knowledge that is critical for understanding brain plasticity (Poldrack, 2000). For example, a functional reorganization of activation may reflect learningrelated changes in connectivity between regions over time, with the result that changes in levels of activation may be due to changing levels of inter-regional influence, perhaps through increases in inhibitory connections (Fletcher et al., 1999). We may also consider the case where the level of activation in a particular region may remain the same throughout practice but its interaction with other areas may change. The role played by multimodal or multifunctional areas such as PFC in a particular task may only be understandable through an examination of their interactions with other areas (McIntosh, 1998).

The analytic techniques of functional and effective connectivity enable the investigation of practice-related changes in regional interactions. Functional connectivity is the analysis of the pattern of correlations among brain regions whereas effective connectivity attempts to identify patterns of influence between nodes in the network responsible for task performance. Effective connectivity analysis therefore incorporates a model of the anatomical connections between nodes of the hypothesised network and regional patterns of functional activations. These analyses are typically implemented using partial least squares (PLS) or eigenimage analysis and structural equation modelling (SEM) techniques, applied to a covariance matrix extracted from the functional neuroimaging data (see McIntosh et al., 1994, 1996; Buchel et al., 1997; McIntosh, 1998, for a detailed discussion of these methods). We conclude that a better approach to the investigation of practice effects will incorporate these analytical techniques, thereby enabling the 
conjoint analysis of changes in activity within specific regions and patterns of connectivity between regions.

\section{Implications and Conclusions}

Authors such as Munte et al. (2002) have lamented that it is far from clear how the mechanisms governing synaptic plasticity at the cellular level, typically revealed in animal studies, are related to the changes in large-scale neuronal networks on the one hand, and cognitive processes on the other. As we have emphasized, connectivity analyses may go some way to shedding light on this question. That is, the analysis of changes in the ways in which brain areas work together, or influence one another, can form the interpretative link between the functional implications of regional changes in activation, presumably driven by the cellular mechanisms demonstrated in animal models, and changes on the level of cognitive and behavioural processes. At present, there exist only a handful of studies examining practice-related changes in functional and effective connectivity (e.g. Buchel et al., 1999; Fletcher et al., 1999; Toni et al., 2002), indicating the potential for the development of research in this area.

One important issue raised by this review, and constituting a possible avenue for future research, concerns the relationship that exists between functional activation changes and changes in neural connectivity within specific functional brain regions (e.g. horizontal connections). As we have emphasized, the ability to investigate connectivity on a micro scale would benefit our understanding of practice effects, in particular with reference to the possibility of differential plasticity in the different task domains. The present level of fMRI available to most researchers (1-3 T) may not provide sufficient spatial resolution to enable estimates of connectivity on the micro scale - i.e. within-area connectivity. Nevertheless, the technology available to researchers is constantly improving and the development of studies using high-field MRI technology may allow researchers to investigate this micro level, enabling us to tackle those questions outlined above. There are other promising techniques that may allow for these types of investigations. One potential route being explored by some researchers is the combination of DTI and functional or effective connectivity analyses. Provision of in vivo anatomical evidence for the models employed in connectivity analyses by DTI will bolster those analyses. Additionally some animal researchers have already attempted to uncover the practice-related within-area connectivity changes using animal models such as the rat (e.g. Rioult-Pedotti et al., 1998, 2000). Further investigations along these lines are necessary in order to develop our understanding of the complex plastic changes arising from task practice.

Already a number of studies have examined practice-related structural changes on a macro level. For example, studies have shown increases in grey and white matter volume in several regions in the brains of highly experienced musicians (see Schlaug, 2001; Munte et al., 2002). More recently, Draganski et al. (2004), using voxel-based morphometry, revealed how transient structural changes in grey matter volume could take place over a relatively short time period ( 3 months) and be associated with the visual processing and storage requirements of the practice of an entirely novel motor skill (juggling). In this study they also observed a relationship between the structural grey-matter changes and juggling performance. Maguire $e t$ al. (2000) have demonstrated similar experience and learningrelated structural change in the hippocampus of London taxi- drivers. Further exploration of the relationship between these structural changes and the functional changes in activation we have reviewed above is clearly warranted.

In conclusion, we have conducted a comprehensive review of the practice effects literature, which has enabled us to draw a number of conclusions concerning the impact of particular factors on the pattern of activation changes that are observed. We have shown how the pattern of practice-related changes seen in any study is explicable by reference to a number of factors that play a role in the task, namely the effect of practice on the cognitive processes involved in task performance, the effect of task domain, the effect of the time-window of imaging, and a number of other miscellaneous and confounding factors. We conclude that it is with a focus on the conjoint analysis of changes in activity within specific regions and patterns of connectivity between regions that our understanding of practice effects will progress most rapidly, and that these analyses will be supported by current and developing imaging tools such as DTI and high-field MRI.

\section{Notes}

Address correspondence to Hugh Garavan, Department of Psychology, Trinity College Dublin, Dublin 2, Ireland. Email: hugh.garavan@ tcd.ie.

\section{References}

Andreasen NC, O’Leary DS, Arndt S, Cizadlo T, Rezai K, Watkins GL, Ponto LL, Hichwa RD (1995a) PET studies of memory I: novel and practiced free recall of complex narratives. Neuroimage 2:284-295.

Andreasen NC, O'Leary DS, Cizadlo T, Arndt S, Rezai K, Watkins GL, Ponto LL, Hichwa RD (1995b) PET studies of memory II: novel versus practiced free recall of word lists. Neuroimage 2:296-305.

Asaad WF, Rainer G, Miller EK (1998) Neural activity in the primate prefrontal cortex during associative learning. Neuron 21:1399-407.

Beauchamp MH, Dagher A, Aston JA, Doyon J (2003) Dynamic functional changes associated with cognitive skill learning of an adapted version of the Tower of London task. Neuroimage 20:1649-1660.

Bernstein LJ, Beig S, Siegenthaler AL, Grady CL (2002) The effect of encoding strategy on the neural correlates of memory for faces. Neuropsychologia 40:86-98.

Buchel C, Friston K (2000) Assessing interactions among neuronal systems using functional neuroimaging. Neural Netw 13:871-882.

Buchel C, Friston KJ (1997) Modulation of connectivity in visual pathways by attention: cortical interactions evaluated with structural equation modelling and fMRI. Cereb Cortex 7:768-778.

Buchel C, Coull JT, Friston KJ (1999) The predictive value of changes in effective connectivity for human learning. Science 283:1538-1541.

Buonomano DV, Merzenich MM (1998) Cortical plasticity: from synapses to maps. Annu Rev Neurosci 21:149-186.

Bush G, Whalen PJ, Rosen BR, Jenike MA, McInerney SC, Rauch SL (1998) The counting Stroop: an interference task specialized for functional neuroimaging — validation study with functional MRI. Hum Brain Mapp 6:270-282.

Chen LL, Wise SP (1995) Neuronal activity in the supplementary eye field during acquisition of conditional oculomotor associations. J Neurophysiol 73:1101-1121.

Debaere F, Wenderoth N, Sunaert S, Van Hecke P, Swinnen SP (2004) Changes in brain activation during the acquisition of a new bimanual coordination task. Neuropsychologia 42:855-867.

Doyon J, Owen AM, Petrides M, Sziklas V, Evans AC (1996) Functional anatomy of visuomotor skill learning in human subjects examined with positron emission tomography. Eur J Neurosci 8:637-648.

Doyon J, Song AW, Karni A, Lalonde F, Adams MM, Ungerleider LG (2002) Experience-dependent changes in cerebellar contributions to motor sequence learning. Proc Natl Acad Sci USA 99: 1017-1022. 
Draganski B, Gaser C, Busch V, Schuierer G, Bogdahn U, May A (2004) Changes in grey matter induced by training. Nature 427:311-312.

Druzgal TJ, D'Esposito M (2003) Dissecting contributions of prefrontal cortex and fusiform face area to face working memory. $\mathrm{J}$ Cogn Neurosci 15:771-784

Duncan J (2001) An adaptive coding model of neural function in prefrontal cortex. Nat Rev Neurosci 2:820-829.

Elbert T, Pantev C, Wienbruch C, Rockstroh B, Taub E (1995) Increased cortical representation of the fingers of the left hand in string players. Science 270:305-307.

Fletcher P, Buchel C, Josephs O, Friston K, Dolan R (1999) Learningrelated neuronal responses in prefrontal cortex studied with functional neuroimaging. Cereb Cortex 9:168-178.

Friston K (2002) Beyond phrenology: what can neuroimaging tell us about distributed circuitry? Annu Rev Neurosci 25:221-250

Frutiger SA, Strother SC, Anderson JR, Sidtis JJ, Arnold JB, Rottenberg DA (2000) Multivariate predictive relationship between kinematic and functional activation patterns in a PET study of visuomotor learning. Neuroimage 12:515-27.

Garavan H, Kelley D, Rosen A, Rao SM, Stein EA (2000) Practice-related functional activation changes in a working memory task. Microsc Res Tech 51:54-63.

Glabus MF, Horwitz B, Holt JL, Kohn PD, Gerton BK, Callicott JH, MeyerLindenberg A, Berman KF (2003) Interindividual differences in functional interactions among prefrontal, parietal and parahippocampal regions during working memory. Cereb Cortex 13:1352-1361.

Grafton ST, Mazziotta JC, Presty S, Friston KJ, Frackowiak RS, Phelps ME (1992) Functional anatomy of human procedural learning determined with regional cerebral blood flow and PET. J Neurosci 12:2542-2548.

Grafton ST, Hazeltine E, Ivry RB (1995) Functional anatomy of sequence learning in normal humans. J Cogn Neurosci 7:497-510.

Haier RJ, Siegel BV, Jr., MacLachlan A, Soderling E, Lottenberg S Buchsbaum MS (1992) Regional glucose metabolic changes after learning a complex visuospatial/motor task: a positron emission tomographic study. Brain Res 570:134-143.

Hazeltine E, Grafton ST, Ivry R (1997) Attention and stimulus characteristics determine the locus of motor-sequence encoding. A PET study. Brain 120:123-140.

Hempel A, Giesel FL, Caraballo NMG, Amann M, Meyer H, Wustenberg T, Essig M, Schroeder J (2004) Plasticity of cortical activation related to working memory during training. Am J Psychiatry 161:745-747.

Hikosaka O, Miyashita K, Miyachi S, Sakai K, Lu X (1998) Differential roles of the frontal cortex, basal ganglia, and cerebellum in visuomotor sequence learning. Neurobiol Learn Mem 70:137-149.

Hikosaka O, Nakahara H, Rand MK, Sakai K, Lu X, Nakamura K, Miyachi S, Doya K (1999) Parallel neural networks for learning sequential procedures. Trends Neurosci 22:464-471.

Hikosaka O, Nakamura K, Sakai K, Nakahara H (2002) Central mechanisms of motor skill learning. Curr Opin Neurobiol 12:217-222.

Honda M, Deiber MP, Ibanez V, Pascual-Leone A, Zhuang P, Hallett M (1998) Dynamic cortical involvement in implicit and explicit motor sequence learning. A PET study. Brain 121:2159-2173.

Hund-Georgiadis M, von Cramon DY (1999) Motor-learning-related changes in piano players and non-musicians revealed by functional magnetic-resonance signals. Exp Brain Res 125:417-425.

Iacoboni M, Woods RP, Mazziotta JC (1996) Brain-behavior relationships: evidence from practice effects in spatial stimulus-response compatibility. J Neurophysiol 76:321-331.

Jansma JM, Ramsey NF, Coppola R, Kahn RS (2000) Specific versus nonspecific brain activity in a parametric N-back task. Neuroimage 12:688-697.

Jansma JM, Ramsey NF, Slagter HA, Kahn RS (2001) Functional anatomical correlates of controlled and automatic processing. J Cogn Neurosci 13:730-743.

Jonides J (2004) How does practice makes perfect? Nat Neurosci 7:10-11.

Jueptner M, Stephan KM, Frith CD, Brooks DJ, Frackowiak RS, Passingham RE (1997) Anatomy of motor learning. I. Frontal cortex and attention to action. J Neurophysiol 77:1313-1324.
Karni A, Meyer G, Jezzard P, Adams MM, Turner R, Ungerleider LG (1995) Functional MRI evidence for adult motor cortex plasticity during motor skill learning. Nature 377:155-158.

Karni A, Meyer G, Rey-Hipolito C, Jezzard P, Adams MM, Turner R, Ungerleider LG (1998) The acquisition of skilled motor performance: fast and slow experience-driven changes in primary motor cortex. Proc Natl Acad Sci USA 95:861-868.

Kassubek J, Schmidtke K, Kimmig H, Lucking CH, Greenlee MW (2001) Changes in cortical activation during mirror reading before and after training: an fMRI study of procedural learning. Brain Res Cogn Brain Res 10:207-217.

Kleim JA, Hogg TM, VandenBerg PM, Cooper NR, Bruneau R, Remple M (2004) Cortical synaptogenesis and motor map reorganization occur during late, but not early, phase of motor skill learning. J Neurosci 24:628-633.

Klingberg T, Forssberg H, Westerberg H (2002) Training of working memory in children with ADHD. J Clin Exp Neuropsychol 24:781-791.

Kolb B, Gibb R (2002) Frontal lobe plasticity and behaviour. In: Principles of frontal lobe function (Stuss DT, Knight RT, eds). London: Oxford University Press.

Kolb B, Whishaw IQ (1998) Brain plasticity and behavior. Annu Rev Psychol 49:43-64.

Korman M, Raz N, Flash T, Karni A (2003) Multiple shifts in the representation of a motor sequence during the acquisition of skilled performance. Proc Natl Acad Sci U S A 100:12492-12497.

Landau SM, Schumacher EH, Garavan H, Druzgal TJ, D’Esposito M (2004) A functional MRI study of the influence of practice on component processes of working memory. Neuroimage 22:211-221.

Maguire EA, Gadian DG, Johnsrude IS, Good CD, Ashburner J, Frackowiak RS, Frith CD (2000) Navigation-related structural change in the hippocampi of taxi drivers. Proc Natl Acad Sci USA 97: 4398-4403.

McIntosh AR (1998) Understanding neural interactions in learning and memory using functional neuroimaging. Ann NY Acad Sci 855: 556-571.

McIntosh AR (1999) Mapping cognition to the brain through neural interactions. Memory 7:523-548.

McIntosh AR, Gonzalez-Lima F (1994) Structual equation modelling and its application to network analysis in functional brain imaging. Hum Brain Mapp 2:2-22.

McIntosh AR, Bookstein FL, Haxby JV, Grady CL (1996) Spatial pattern analysis of functional brain images using partial least squares. Neuroimage 3:143-157.

McIntosh AR, Rajah MN, Lobaugh NJ (1999) Interactions of prefrontal cortex in relation to awareness in sensory learning. Science 284:1531-1533.

Meegan DV, Purc-Stephenson R, Honsberger MJ, Topan M (2004) Task analysis complements neuroimaging: an example from working memory research. Neuroimage 21:1026-1036.

Miller EK, Cohen JD (2001) An integrative theory of prefrontal cortex function. Annu Rev Neurosci 24:167-202.

Munte TF, Altenmuller E, Jancke L (2002) The musician's brain as a model of neuroplasticity. Nat Rev Neurosci 3:473-8.

Neubauer AC, Grabner RH, Freudenthaler HH, Beckmann JF, Guthke J (2004) Intelligence and individual differences in becoming neurally efficient. Acta Psychol (Amst) 116:55-74.

Nezafat R, Shadmehr R, Holcomb HH (2001) Long-term adaptation to dynamics of reaching movements: a PET study. Exp Brain Res 140:66-76.

Olesen PJ, Westerberg H, Klingberg T (2004) Increased prefrontal and parietal activity after training of working memory. Nat Neurosci 7:75-79.

Pantev C, Oostenveld R, Engelien A, Ross B, Roberts LE, Hoke M (1998) Increased auditory cortical representation in musicians. Nature 392:811-814.

Pascual-Leone A, Torres F (1993) Plasticity of the sensorimotor cortex representation of the reading finger in Braille readers. Brain 116:39-52.

Petersen SE, van Mier H, Fiez JA, Raichle ME (1998) The effects of practice on the functional anatomy of task performance. Proc Natl Acad Sci USA 95:853-860. 
Petersson KM, Elfgren C, Ingvar M (1999) Dynamic changes in the functional anatomy of the human brain during recall of abstract designs related to practice. Neuropsychologia 37:567-587.

Poldrack RA (2000) Imaging brain plasticity: conceptual and methodological issues-a theoretical review. Neuroimage 12:1-13.

Poldrack RA, Desmond JE, Glover GH, Gabrieli JD (1998) The neural basis of visual skill learning: an fMRI study of mirror reading. Cereb Cortex 8:1-10.

Poldrack RA, Gabrieli JD (2001) Characterizing the neural mechanisms of skill learning and repetition priming: evidence from mirror reading. Brain 124:67-82.

Raichle ME, Fiez JA, Videen TO, MacLeod AM, Pardo JV, Fox PT, Petersen SE (1994) Practice-related changes in human brain functional anatomy during nonmotor learning. Cereb Cortex 4: 8-26.

Raichle ME, MacLeod AM, Snyder AZ, Powers WJ, Gusnard DA, Shulman GL (2001) A default mode of brain function. Proc Natl Acad Sci USA 98:676-82.

Rainer G, Asaad WF, Miller EK (1998) Selective representation of relevant information by neurons in the primate prefrontal cortex. Nature 393:577-579.

Ramsey NF, Jansma JM, Jager G, Van Raalten T, Kahn RS (2004) Neurophysiological factors in human information processing capacity. Brain 127:517-525.

Rioult-Pedotti MS, Friedman D, Hess G, Donoghue JP (1998) Strengthening of horizontal cortical connections following skill learning. Nat Neurosci 1:230-234.

Rioult-Pedotti MS, Friedman D, Donoghue JP (2000) Learning-induced LTP in neocortex. Science 290:533-536.

Rypma B, D'Esposito M (1999) The roles of prefrontal brain regions in components of working memory: effects of memory load and individual differences. Proc Natl Acad Sci USA 96: 6558-6563.

Rypma B, Berger JS, D'Esposito M (2002) The influence of workingmemory demand and subject performance on prefrontal cortical activity. J Cogn Neurosci 14:721-731.

Sakai K, Hikosaka O, Miyauchi S, Takino R, Sasaki Y, Putz B (1998) Transition of brain activation from frontal to parietal areas in visuomotor sequence learning. J Neurosci 18:1827-1840.
Sanes JN, Donoghue JP (2000) Plasticity and primary motor cortex. Annu Rev Neurosci 23:393-415.

Schiltz C, Bodart JM, Dubois S, Dejardin S, Michel C, Roucoux A, Crommelinck M, Orban GA (1999) Neuronal mechanisms of perceptual learning: changes in human brain activity with training in orientation discrimination. Neuroimage 9:46-62.

Schiltz C, Bodart JM, Michel C, Crommelinck M (2001) A pet study of human skill learning: changes in brain activity related to learning an orientation discrimination task. Cortex 37:243-265.

Schlaug G (2001) The brain of musicians. A model for functional and structural adaptation. Ann NY Acad Sci 930:281-299.

Seidler RD, Purushotham A, Kim SG, Ugurbil K, Willingham D, Ashe J (2002) Cerebellum activation associated with performance change but not motor learning. Science 296:2043-2046.

Shadmehr R, Holcomb HH (1997) Neural correlates of motor memory consolidation. Science 277:821-5.

Toni I, Krams M, Turner R, Passingham RE (1998) The time course of changes during motor sequence learning: a whole-brain fMRI study. Neuroimage 8:50-61.

Toni I, Ramnani N, Josephs O, Ashburner J, Passingham RE (2001) Learning arbitrary visuomotor associations: temporal dynamic of brain activity. Neuroimage 14:1048-1057.

Toni I, Rowe J, Stephan KE, Passingham RE (2002) Changes of corticostriatal effective connectivity during visuomotor learning. Cereb Cortex 12:1040-1047.

Ungerleider LG, Doyon J, Karni A (2002) Imaging brain plasticity during motor skill learning. Neurobiol Learn Mem 78:553-564.

van Mier H, Tempel LW, Perlmutter JS, Raichle ME, Petersen SE (1998) Changes in brain activity during motor learning measured with PET: effects of hand of performance and practice. J Neurophysiol 80: 2177-2199.

van Turennout M, Bielamowicz L, Martin A (2003) Modulation of neural activity during object naming: effects of time and practice. Cereb Cortex 13:381-391.

Weissman DH, Woldorff MG, Hazlett CJ, Mangun GR (2002) Effects of practice on executive control investigated with fMRI. Brain Res Cogn Brain Res 15:47-60.

Wolpaw JR (1997) The complex structure of a simple memory. Trends Neurosci 20:588-594. 